05,12

\title{
Динамика импульсного перемагничивания магнитоодноосных наночастиц
}

\author{
(c) А.М. Шутый, Д.И. Семенцов \\ Ульяновский государственный университет, \\ Ульяновск, Россия \\ E-mail: shuty@mail.ru \\ Поступила в Редакцию 7 апреля 2019 г. \\ В окончательной редакции 5 мая 2019 г. \\ Принята к публикации 14 мая 2019 г.
}

\begin{abstract}
Исследован отклик магнитного момента магнитоодноосной наночастицы и плоской решетки из подобных наночастиц на действие короткого гауссова импульса магнитного поля при наличии и в отсутствие его модуляции. Выявлена и проанализирована периодическая зависимость от длительности импульса и его пикового значения продолжительности отклика и конечной ориентации магнитных моментов. Исследовано влияние на процессы перемагничивания слабого подмагничивающего поля и отклонения поля импульса от поперечной ориентации. Показано, что влияние диполь-дипольного взаимодействия приводит к модуляции отклика на импульсное воздействие.
\end{abstract}

Ключевые слова: импульсное перемагничивание; прецессионная динамика; одноосная анизотропия; магнитная наночастица.

DOI: 10.21883 /FTT.2019.10.48249.448

\section{1. Введение}

В последние десятилетия достигнут значительный прогресс в понимании свойств и динамических процессов в системах магнитных наночастиц [1-7]. Благодаря малому размеру, магнитные наночастицы, как правило, однодоменны, что позволяет упростить описание структур на их основе. Основной вклад во взаимодействие наночастиц вносит диполь-дипольное взаимодействие, которое определяется не только их собственным магнитным моментом, но и локальным упорядочением частиц, а также магнитной анизотропией [8-11]. Дискретность решеточных структур наночастиц приводит к существенным отличиям равновесных состояний и динамического перемагничивания от аналогичных свойств макроскопических монодоменных объектов [12-14]. К таким отличиям, например, могут быть отнесены бистабильные состояния решеток, обусловленные наличием различных равновесных ориентационных конфигураций.

Возможность записи информации на решетках магнитных диполей основана на изменении равновесной конфигурации магнитных моментов за счет воздействия импульсов магнитного поля $[15,16]$. По этой причине импульсному перемагничиванию магнитных микро- и наносистем посвящено значительное число как теоретических, так и экспериментальных работ [17-26]. Так, в работе [17] экспериментально была обнаружена периодичность реализации перемагничивания планарной слоистой микроструктуры при изменении длительности и амплитуды импульса, что объяснено доминирующей ролью фазовой когерентности между прецессией намагниченности и импульсом поля во время переключения. В [18] экспериментально исследуется прецессионный отклик намагниченности микроскопической ячейки памяти на импульсное воздействие, при этом выявлены короткие по времени переключения ячейки, когда после затухания импульса поля подавляются длинноволновые магнитные возбуждения. В работах [19-22] исследована динамика намагниченности антиферромагнитных систем под влиянием сверхбыстрых импульсов магнитного поля. В [19], в частности, продемонстрирована возможность перемагничивания системы с длительным релаксационным процессом, а в [22] показана возможность перемагничивания без длительного релаксационного процесса при использовании специальной формы полевого сигнала. Основываясь на уравнении Ландау-Лифшица, в [23] рассмотрено переключение намагниченности монодоменных наночастиц и предложена схема для ее приведения в заданное состояние с помощью ультракоротких магнитных импульсов. В работах [24-26] исследуется импульсное перемагничивание пленок с различным типом магнитной анизотропии, а также рассматриваются динамические петли гистерезиса и релаксационные эффекты при импульсном перемагничивании наночастиц.

В настоящей работе на основе численного решения динамических уравнений исследуется отклик на гауссов импульс магнитного поля магнитного момента изолированной наночастицы, обладающей одноосной анизотропией, и плоской решетки подобных наночастиц. Выявляются условия импульсного перемагничивания диполей и влияние на динамику отклика диполь-дипольного взаимодействия между элементами решетки. Рассмотрение широкого интервала значений длительности и пикового значения импульса и построение соответствующих диаграмм позволило выявить периодические зависимости от параметров импульса как продолжительности 
прецессионного отклика магнитного момента, так и реализации перемагничивания наночастиц. Исследовано влияние на процессы импульсного перемагничивания наночастиц слабого статического поля, отклонения поля импульса от поперечной ориентации, а также модуляции действующего импульса.

\section{2. Исходные уравнения}

Рассмотрим плоский массив $6 \times 6$ идентичных наночастиц с магнитным моментом $\left|\mathbf{m}_{i}\right|=m$ и формой, близкой к сферической. Считаем, что наночастицы расположены в узлах квадратной решетки с параметром решетки $r_{0}$, каждая из них обладает одноосной магнитной анизотропией и имеет размер, при котором находится в однодоменном состоянии. Энергию $i$-ой наночастицы запишем в виде суммы зеемановской энергии во внешнем магнитном поле $\mathbf{H}$, энергии диполь-дипольного взаимодействия и энергий анизотропии

$$
W\left(\mathbf{m}_{i}\right)=-\mathbf{m}_{i} \mathbf{H}+W_{d}\left(\mathbf{m}_{i}, \mathbf{m}_{n}\right)+W_{a}\left(\mathbf{m}_{i}\right) .
$$

Здесь внешнее магнитное поле является суммой статического и высокочастотного полей. Энергия диполь-дипольного взаимодействия

$$
W_{d}\left(\mathbf{m}_{i}, \mathbf{m}_{n}\right)=\sum_{n \neq i}\left(\frac{\mathbf{m}_{i} \mathbf{m}_{n} r_{i n}^{2}-3\left(\mathbf{m}_{i} \mathbf{r}_{i n}\right)\left(\mathbf{m}_{n} \mathbf{r}_{i n}\right)}{r_{i n}^{5}}\right),
$$

где $\mathbf{r}_{i n}$ и $r_{i n}-$ радиус-вектор и расстояние между $i$-ым и $n$-ым диполями. Энергия одноосной анизотропии определяется выражением

$$
W_{a}\left(\mathbf{m}_{i}\right)=-K_{u} V_{0} \frac{\left(\mathbf{m}_{i} \mathbf{n}\right)^{2}}{m_{i}^{2}},
$$

где $K_{u}$ и $\mathbf{n}$ - константа одноосной анизотропии и орт оси легкого намагничивания (ОЛН), $V_{0}$ - объем наночастицы.

Динамика каждого из моментов дипольной решетки описывается уравнением Ландау-Лифшица с релаксационным членом в форме Гильберта [27]:

$$
\frac{\partial \mathbf{m}_{i}}{\partial t}=-\gamma \mathbf{m}_{i} \times \mathbf{H}_{i}^{\mathrm{eff}}-\frac{\alpha}{m_{i}} \mathbf{m}_{i} \times \frac{\partial \mathbf{m}_{i}}{\partial t},
$$

где $\gamma$ - гиромагнитное отношение, $\alpha$ - параметр диссипации. Эффективное магнитное поле, создаваемое в месте расположения $i$-го диполя остальными диполями и внешним полем $\mathbf{H}$, с учетом (1) имеет вид

$$
\begin{aligned}
\mathbf{H}_{i}^{\mathrm{eff}}=-\frac{\partial W_{i}}{\partial \mathbf{m}_{i}}=\mathbf{H} & +K_{u} V_{0} \frac{\mathbf{n}\left(\mathbf{m}_{i} \mathbf{n}\right)}{m_{i}^{2}} \\
& +\sum_{n \neq i} \frac{3\left(\mathbf{m}_{n} \mathbf{r}_{i n}\right) \mathbf{r}_{i n}-\mathbf{m}_{n} r_{i n}^{2}}{r_{i n}^{5}} .
\end{aligned}
$$

Далее перейдем к безразмерным параметрам: где $\boldsymbol{\mu}_{i}=\mathbf{m}_{i} / m, \quad \mathbf{e}_{i n}=\mathbf{r}_{i n} / r_{i n}, \quad \tau=\gamma J t, \quad l_{i n}^{3}=r_{i n}^{3} / V_{0}, \quad$ где
$J=m / V_{0}-$ намагниченность наночастицы. Безразмерный параметр решетки, то есть расстояние между центрами ближайших наночастиц, $\rho=r_{0} V_{0}^{-1 / 3}$. В безразмерных параметрах уравнения (4) принимают вид

$$
\frac{\partial \boldsymbol{\mu}_{i}}{\partial \tau}=-\boldsymbol{\mu}_{i} \times \mathbf{h}_{i}^{\mathrm{eff}}-\alpha \boldsymbol{\mu}_{i} \times \frac{\partial \boldsymbol{\mu}_{i}}{\partial \tau}
$$

где

$$
\mathbf{h}_{i}^{\mathrm{eff}}=\mathbf{h}+k_{u} \mathbf{n}\left(\boldsymbol{\mu}_{i} \mathbf{n}\right)+\sum_{n \neq i}\left[\frac{3\left(\boldsymbol{\mu}_{n} \mathbf{e}_{i n}\right) \mathbf{e}_{i n}-\boldsymbol{\mu}_{n}}{l_{i n}^{3}}\right] .
$$

В этом случае обезразмеренные внешнее поле и константа одноосной анизотропии принимают вид: $\mathbf{h}=\mathbf{H} / J$, $k_{u}=K_{u} / J^{2}$.

Приведем переход от безразмерных величин к размерным для дипольной решетки наночастиц, состоящих из $N$ атомов железа: магнитный момент наночастицы $m \approx 2.2 \mu_{B}$, где $\mu_{B}-$ магнетон Бора. Так, для устойчивой сферической конфигурации $N=561$ и радиус наночастицы составляет $R=1.364 \cdot 10^{-7} \mathrm{~cm}$, а $m \approx 1.145 \cdot 10^{-17} \mathrm{erg} / \mathrm{Oе}$ и $J \approx 1.08 \mathrm{kG}$. С учетом $\gamma=1.76 \cdot 10^{7}(\mathrm{Oe} \cdot \mathrm{s})^{-1}$ получаем следующие численные оценки для времени $t=\tau /(\gamma J) \approx 0.53 \tau \mathrm{ps,} \mathrm{магнитно-}$ го поля $H=J h \approx 1.08 h \mathrm{kOe}$ и константы анизотропии $K_{u}=J^{2} k_{u} \approx 1.2 \cdot 10^{6} k_{u} \mathrm{erg} / \mathrm{cm}^{3}$.

При дальнейшем анализе векторное уравнение (6) представляется тремя скалярными уравнениями. Так, для $x$-компонент $\partial \boldsymbol{\mu}_{i} / \partial \tau$ получаем

$$
\begin{aligned}
\left(1+\alpha^{2}\right) & \frac{\partial \mu_{i x}}{\partial \tau}=\left(\mu_{i z}+\alpha \mu_{i x} \mu_{i y}\right) h_{i y}^{\mathrm{eff}} \\
& \quad-\left(\mu_{i y}-\alpha \mu_{i z} \mu_{i x}\right) h_{i z}^{\mathrm{eff}}-\alpha\left(1-\mu_{i x}^{2}\right) h_{i x}^{\mathrm{eff}} .
\end{aligned}
$$

Уравнения для остальных компонент имеют аналогичный вид и могут быть получены циклической перестановкой составляющих.

Далее будет рассмотрена как изолированная наночастица, так и решетка идентичных наночастиц $6 \times 6$. Система координат выбрана таким образом, что ось $X$ перпендикулярна плоскости решетки, а две другие оси параллельны сторонам решетки. Направление ОЛН совпадает с осью $Y$, константа анизотропии принимается равной $k_{u}=1$. Параметр диссипации принимается равным $\alpha=-0.01$. Равновесные ориентации и прецессионные динамические режимы суммарного магнитного момента решетки определяются на основе численного анализа, который проводится с помощью метода Рунге-Кутта.

\section{3. Отклик магнитного момента на импульс магнитного поля}

Рассмотрим отклик изолированной наночастицы на гауссов импульс магнитного поля

$$
h(\tau)=h_{0} \exp \left[-\left(\tau-\tau_{i}\right)^{2} / 2 \tau_{0}^{2}\right],
$$

где $h_{0}, \tau_{i}$ и $\tau_{0}$ - пиковое значение поля, временной сдвиг максимума импульса и его длительность; далее 
принимается $\tau_{i}=200$. В случае отсутствия подмагничивающего поля или при относительно слабом поле $\left(h_{y} \ll k_{u}\right)$, направленном вдоль ОЛН, с изменением длительности (или пикового значения) импульса периодически выполняются условия реализации перемагничивания наночастицы.

На рис. 1 для поляризованного вдоль оси $Y$ подмагничивающего поля $h_{y}=0.1,0,-0.1(a-c)$ приведены диаграммы, отражающие зависимость реализации перемагничивания от амплитуды и длительности гауссова импульса, на которых затемненные области соответствуют осуществлению перемагничивания при данных параметрах импульса, а незатемненные области - отсутствию перемагничивания. В исходном состоянии магнитный момент наночастицы ориентирован в положительном направлении оси $Y$. Из диаграмм видно, что периодичность реализации перемагничивания имеет место как при изменении длительности импульса, так и при изменении его амплитуды, и период соответствующих интервалов уменьшается с возрастанием величины параметров. При этом в отсутствие подмагничивающего поля $(b)$ при достаточно больших параметрах импульса имеет место приближенное равенство между интервалами, отвечающими перемагничиванию и его отсутствию. При слабом подмагничивающем поле, сонаправленном магнитному моменту $(a)$, увеличивается период рассматриваемых интервалов, становятся ограниченными число интервалов, отвечающих перемагничиванию, и область значений длительности импульса, в которой возможно осуществление перемагничивания наночастицы. При слабом подмагничивающем поле, направленном противоположно исходной ориентации магнитных моментов $(c)$, наблюдается относительное сужение интервалов, отвечающих неперемагничиванию системы, а область значений длительности импульса, в которой перемагничивание отсутствует, также становится ограниченной. Аналогичные диаграммы были также представлены в работе [17] для описания данных эксперимента по импульсному перемагничиванию магнито-анизотропной пленочной микроячейки.

Дополнительные исследования показали, что возникающее при условии $h_{0}>k_{u}$ (в отсутствии подмагничивающего поля) чередование интервалов перемагничивания/неперемагничивания имеет место и в случае ступенчатого импульса поля. Для выявления общих закономерностей рассмотрим более сложные профили импульсов, а именно, гармонически модулированные короткие гауссовы импульсы:

$$
h(\tau)=h_{0} \cos (\Omega \tau) \exp \left[-\left(\tau-\tau_{i}\right)^{2} / 2 \tau_{0}^{2}\right],
$$

где $\Omega$ - безразмерная частота модуляции импульса.

На рис. 2 приведены диаграммы зависимости $Y$-компоненты магнитного момента наночастицы после релаксации от амплитуды импульса при его длительности $\tau_{0}=10$ и частоте модуляции $\Omega=0,0.05,0.07,0.09$, $0.1,0.11,0.2,0.3$ (диаграммы 1-8) - вид импульсов
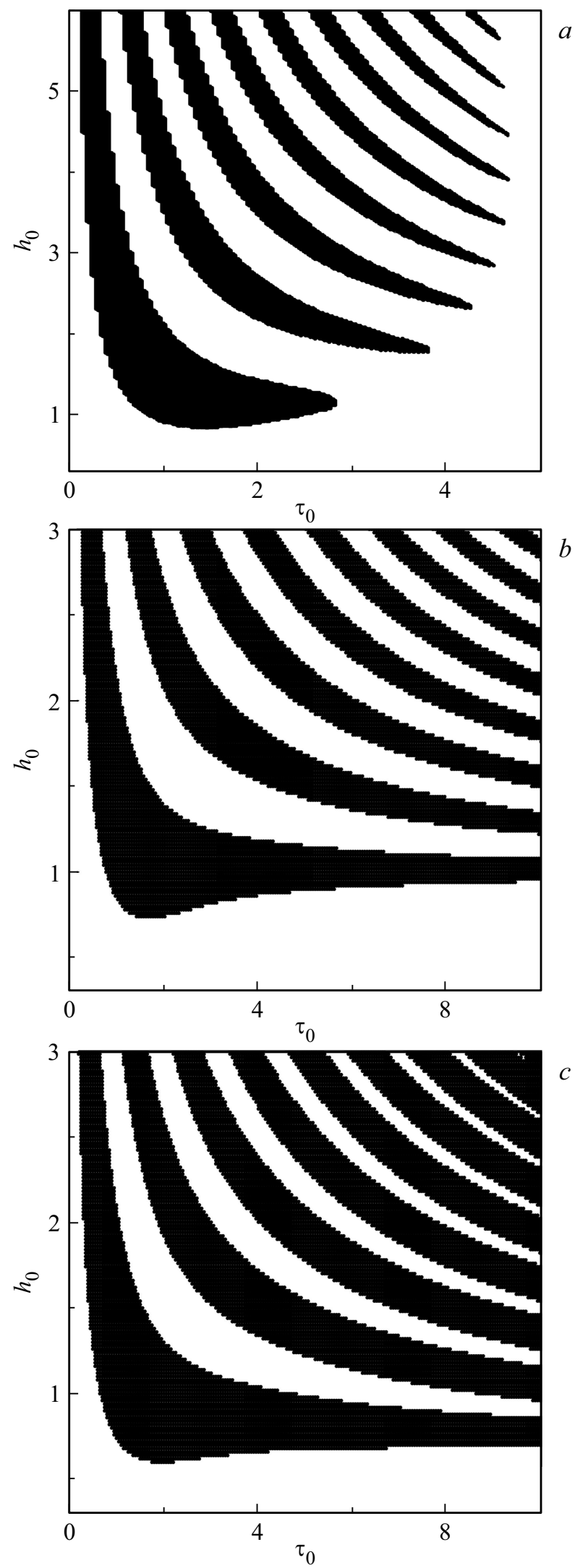

Рис. 1. Диаграммы зависимости реализации перемагничивания наночастицы от амплитуды и длительности импульса при подмагничивающем поле $h_{y}=0.1,0,-0.1(a-c)$ и константе анизотропии $k_{u}=1$; темные области соответствуют перемагничиванию наночастицы. 

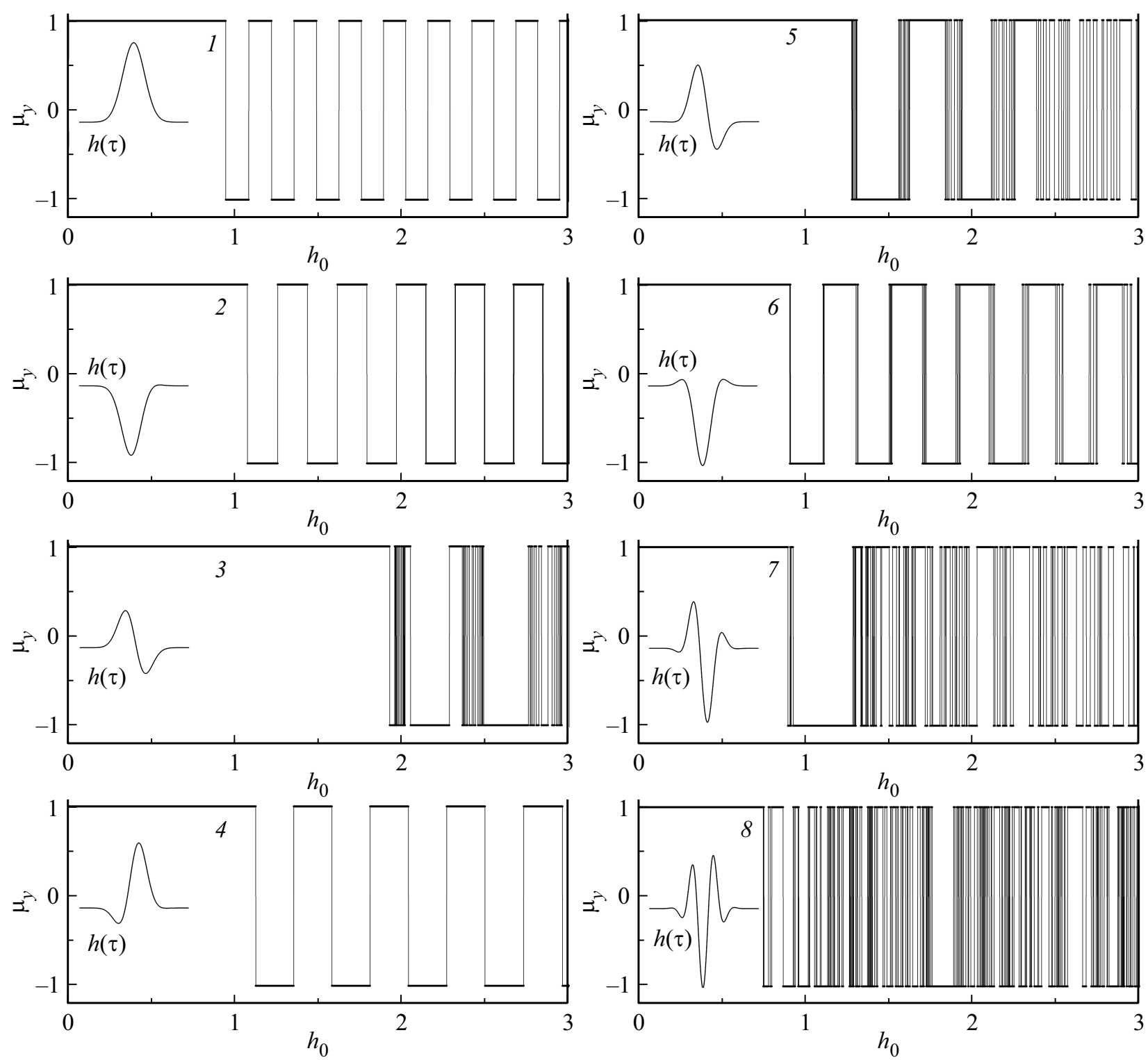

Рис. 2. Диаграммы зависимости перемагничивания наночастицы от амплитуды гауссова импульса при частоте его модуляции $Q=0,0.05,0.07,0.09,0.1,0.11,0.2,0.3(1-8)$ и $\tau_{0}=10-$ вид импульсов приводится слева от диаграмм; интервалы с $\mu_{y}=1$ отвечают отсутствию перемагничивания, с $\mu_{y}=-1-$ реализации перемагничивания, близкое расположение вертикальных ограничительных линий, отвечает бистабильности.

приводится слева от диаграмм. Так как исходным состоянием магнитного момента является $\mu_{y}=1, \mu_{x}=\mu_{z}=0$, то данные диаграммы выявляют интервалы длительности импульса, отвечающие отсутствию перемагничивания $\left(\mu_{y}=1\right)$ и реализации 180-градусного перемагничивания магнитного момента $\left(\mu_{y}=-1\right)$. В случае получения при численном моделировании очень коротких „интервалов“, то есть при близком расположении на диаграммах вертикальных ограничительных линий, имеет место бистабильность, когда после действия импульса магнитный момент может как вернуться в исходное положение, так и поменять направление на противоположное (на реализацию того или иного исхода влияют флуктуации параметров системы). Из диаграмм видно, что отклик на немодулированный импульс (1) характеризуется наиболее узкими интервалами перемагничивания/неперемагничивания и полным отсутствием бистабильности. В случае значительного преобладания одной полуволны модуляции импульса $(2,4,6)$ также наблюдается близкое к периодическому чередование интервалов перемагничивания/неперемагничивания, а бистабильность либо отсутствует, либо имеет место в относительно узких областях вблизи границ интервалов. В остальных случаях модулированного импульса интервалы бистабильности становятся преобладающими, увеличивающимися с ростом амплитуды импульса $(3,5)$, и при увеличении частоты модуляции сливаются в широкие зоны бистабильности. 
На рис. 3 приведена зависимость от времени $Y$-компоненты магнитного момента после действия импульса с параметрами: длительностью $\tau_{0}=10$, частотой модуляции $\Omega=0.1$ и амплитудой $h_{0}=2$ (кривая 1 ), (кривые 2 и 3). Параметры, выбранные для первого случая, отвечают отсутствию бистабильности. При этом реализуется быстрое перемагничивание наночастицы (за время $\Delta \tau<60)$. Во втором и третьем случаях имеет место бистабильность - наночастица может как перемагнититься, так и сохранить исходную ориентацию. Продолжительность отклика при этом большая $(\Delta \tau \geq 500)$. При численном моделировании проявление бистабильности было реализовано малым сдвигом по времени (на единицу) максимума огибающей импульса, что равносильно флуктуации начальных условий.

Процессы перемагничивания сильно зависят от направления поля импульса. На рис. 4. приведены диаграммы зависимости равновесного значения $Y$-компоненты магнитного момента наночастицы от амплитуды гауссова немодулированного (здесь и далее) импульса с $\tau_{0}=10$, когда направление поля импульса ориентировано по отношению к ОЛН (оси $Y$ ) под углом, близким к нормали: $\varphi=89,88,92,100 \mathrm{deg}$ (диаграммы $1-4$ соответственно). Видно, что уже при отклонении от нормали на один градус к направлению исходной ориентации магнитного момента, интервалы, отвечающие перемагничиванию наночастицы, заметно сужаются, а при отклонении на два градуса перемагничивание осуществляется только в четырех очень узких интервалах значения амплитуды импульса. В случае отклонения поля в про-

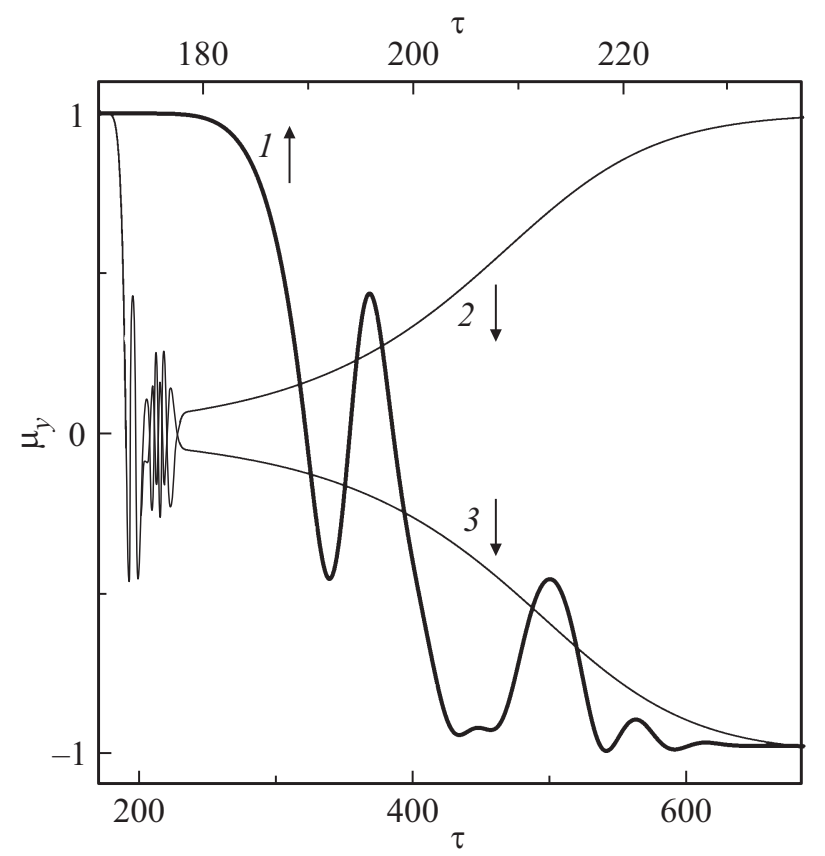

Pис. 3. Зависимость от времени $Y$-компоненты магнитного момента при действии импульса с $\tau_{0}=10, h_{0}=2(1)$, $h_{0}=2.2$ (2 и 3 ) и частотой модуляции $Q=0.1$; кривые 2 и 3 отвечают бистабильности, которая реализована малым сдвигом максимума огибающей импульса: $\tau_{i}=200$ (для кривой 2) и $\tau_{i}=201$ (для кривой 1 ); параметр диссипации $a=-0.01$.
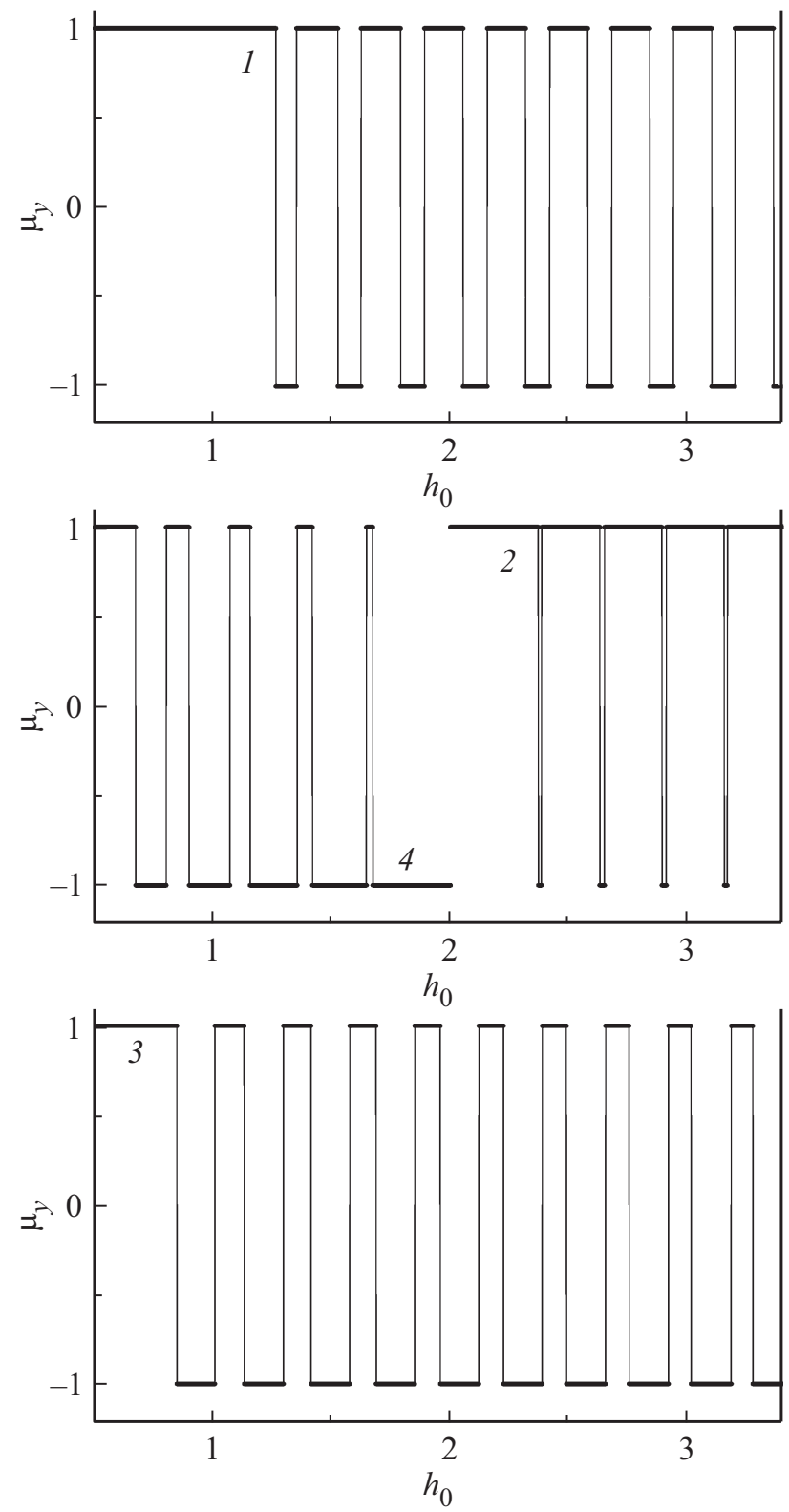

Рис. 4. Диаграммы зависимости перемагничивания наночастицы от амплитуды гауссова импульса с $\tau_{0}=10$ при направлении поля импульса к оси анизотропии под углом $\varphi=89,88,92$, $100 \mathrm{deg}$ (диаграммы $1-4$ ).

тивоположную сторону аналогичные изменения происходят при несколько больших углах: при отклонении на два градуса заметно сужаются интервалы, отвечающие неперемагничиванию наночастицы, а при отклонении на $10 \mathrm{deg}$ только в четырех интервалах (за исключением прилегающего к нулевому значению) магнитный момент возвращается к исходному направлению.

\section{4. Продолжительность отклика}

Прецессионная динамика магнитного момента после действия импульса поля также сложным образом зави- 

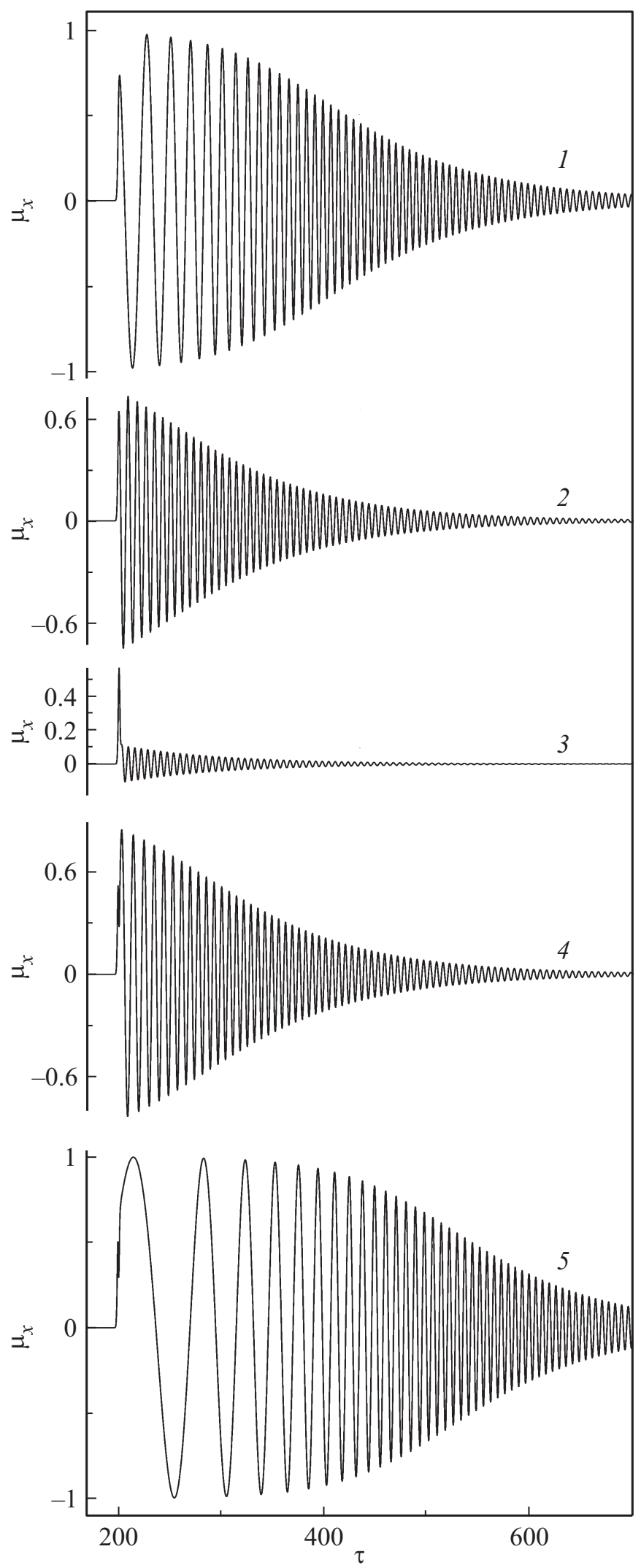

Рис. 5. Зависимость от времени $X$-компонент магнитного момента наночастицы с $k_{u}=1$ при действии импульса с $\tau_{0}=1$ и значениях $h_{0}=0.9,1.1,1.5,1.9,2.1$ (кривые $1-5$ ) - значениях, отвечающих передвижению от одной границы интервала перемагничивания, к другой.
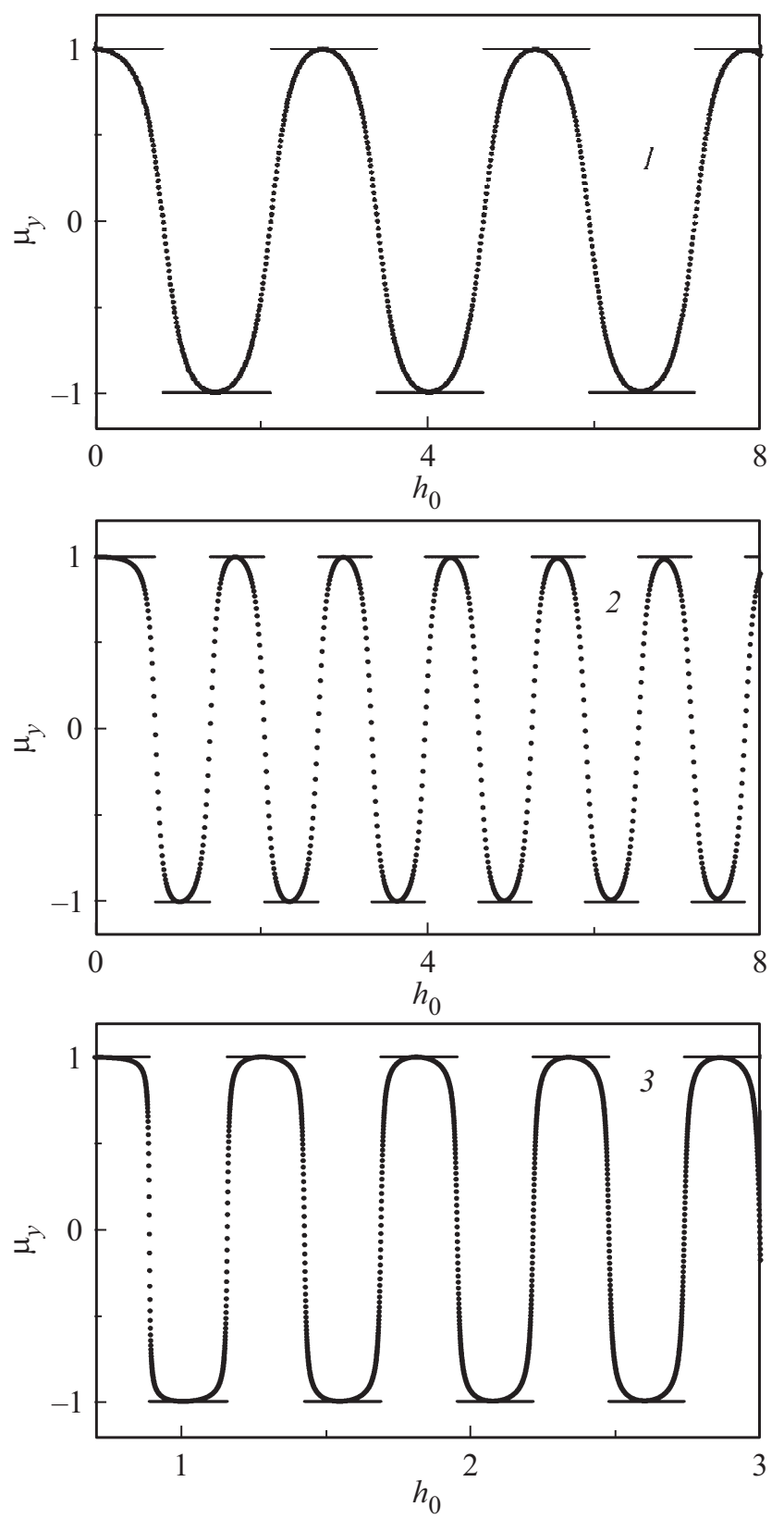

Рис. 6. Диаграммы зависимости от амплитуды импульса с $\tau_{i}=200$ и $0=1,2,5$ (диаграммы $1-3$ ) $Y$-компоненты магнитного момента в момент времени $\tau=250$; интервалам перемагничивания/неперемагничивания наночастицы соответствуют нижние/верхние горизонтальные отрезки.

сит от параметров импульса. На рис. 5 приведена зависимость от времени $X$-компонент магнитного момента наночастицы при действии гауссова импульса с $\tau_{0}=1$ и амплитудой $h_{0}=0.9,1.1,1.5,1.9,2.1$ (кривые 1-5). Выбранные параметры импульса соответствуют передвижению от левой границы интервала $h_{0}$, отвечающего перемагничиванию наночастицы, к правой его границе (при переходе от кривой 1 к 5). Из рисунка видно, что вблизи границ интервала (кривые 1 и 5 ) прецессионный отклик магнитного момента на импульсное воздействие 
имеет большую амплитуду и на два порядка более продолжительный, чем отклик при параметрах импульса, отвечающих центральной области данного интервала
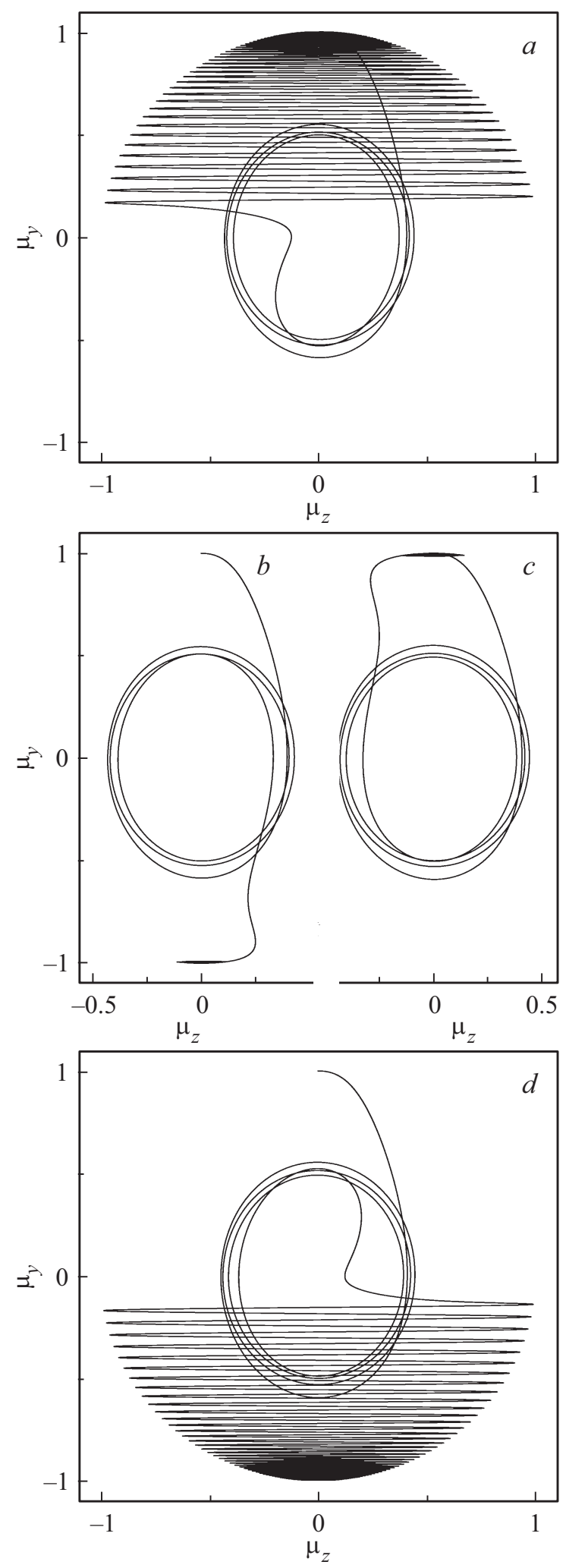

Pис. 7. Проекции траекторий магнитного момента наночастицы на плоскость $Y Z$ при действии импульса с $\tau_{0}=5$ и $h_{0}=2.74,2.6,2.85,3$ (кривые $a-d$ ). (кривая 3). Аналогичные зависимости от длительности импульса отклика магнитного момента изолированной наночастицы, как для анизотропного, так и для изотропного случаев, исследованы в работе [28].

На рис. 6 приведены диаграммы, определяющие зависимость от амплитуды импульса $Y$-компоненты магнитного момента для времени $\tau=250$ при длительности импульса $\tau_{0}=1,2,5$ (диаграммы $1-3$ ). Приведены также диаграммы, выявляющие интервалы перемагничивания/неперемагничивания наночастицы (нижние/верхние горизонтальные отрезки). Из диаграмм видно, что в центральных областях интервалов импульсного параметра, отвечающих как перемагничиванию, так и неперемагничиванию наночастицы, магнитный момент быстро приходит к конечному направлению $(Y$ или $-Y$, в зависимости от интервала значений $\left.h_{0}\right)$, так как уже при выбранном времени $(\tau=250) Y$-компонента приближенно равна \pm 1 . В результате отклик магнитного момента на импульсное воздействие оказывается коротким. Вблизи же границ указанных интервалов при $\tau=250$ величина $\left|\mu_{y}\right| \ll 1$, следовательно, совершаемая после воздействия импульса прецессия магнитного момента в плоскости $X Z$ имеет в данный момент времени большую амплитуду, и отклик на импульс является продолжительным. Из диаграмм также видно, что с увеличением длительности импульса, вместе с сокращением определяющих перемагничивание интервалов, форма рассматриваемых диаграмм приближается к ступенчатому виду. Это означает, что происходит относительное сокращение приграничных областей, отвечающих продолжительному отклику магнитного момента, и в большей части интервалов отклик на импульсное воздействие оказывается коротким.

Для пояснения выявленных эффектов на рис. 7 представлены проекции траекторий магнитного момента на плоскость $Y Z$ при действии импульса длительностью $\tau_{0}=5$ и амплитудой $h_{0}=2.74,2.6,2.85,3$ (кривые $a-d)$. Значения $h_{0}=2.6,2.85$ находятся в центральных областях интервалов, отвечающих перемагничиванию и неперемагничиванию наночастицы, а значения $h_{0}=2.74,3$ располагаются вблизи границы интервалов. Если действие импульса прекращается, когда $Y$-компонента магнитного момента мала $(a$ и $d)$, то под действием поля анизотропии магнитный момент начинает прецессировать, приближаясь к оси $Y$. В результате реализуется большой по продолжительности отклик магнитного момента. Если же действие импульса заканчивается, когда $Y$-компонента близка к значению $\pm 1(b$ и $c)$, прецессионного движения под действием поля анизотропии почти не возникает, и отклик оказывается коротким. Обозначив положительное и отрицательное направления оси $Y$ „исходным“ и „противоположным“ „полюсами“ конфигурации, а плоскость $X Z$ „экваториальной“, можно сказать следующее. Продолжительный отклик без перемагничивания имеет место, когда по прекращении действия импульса магнитный момент ориентирован вблизи экваториальной плоскости на стороне исходного 

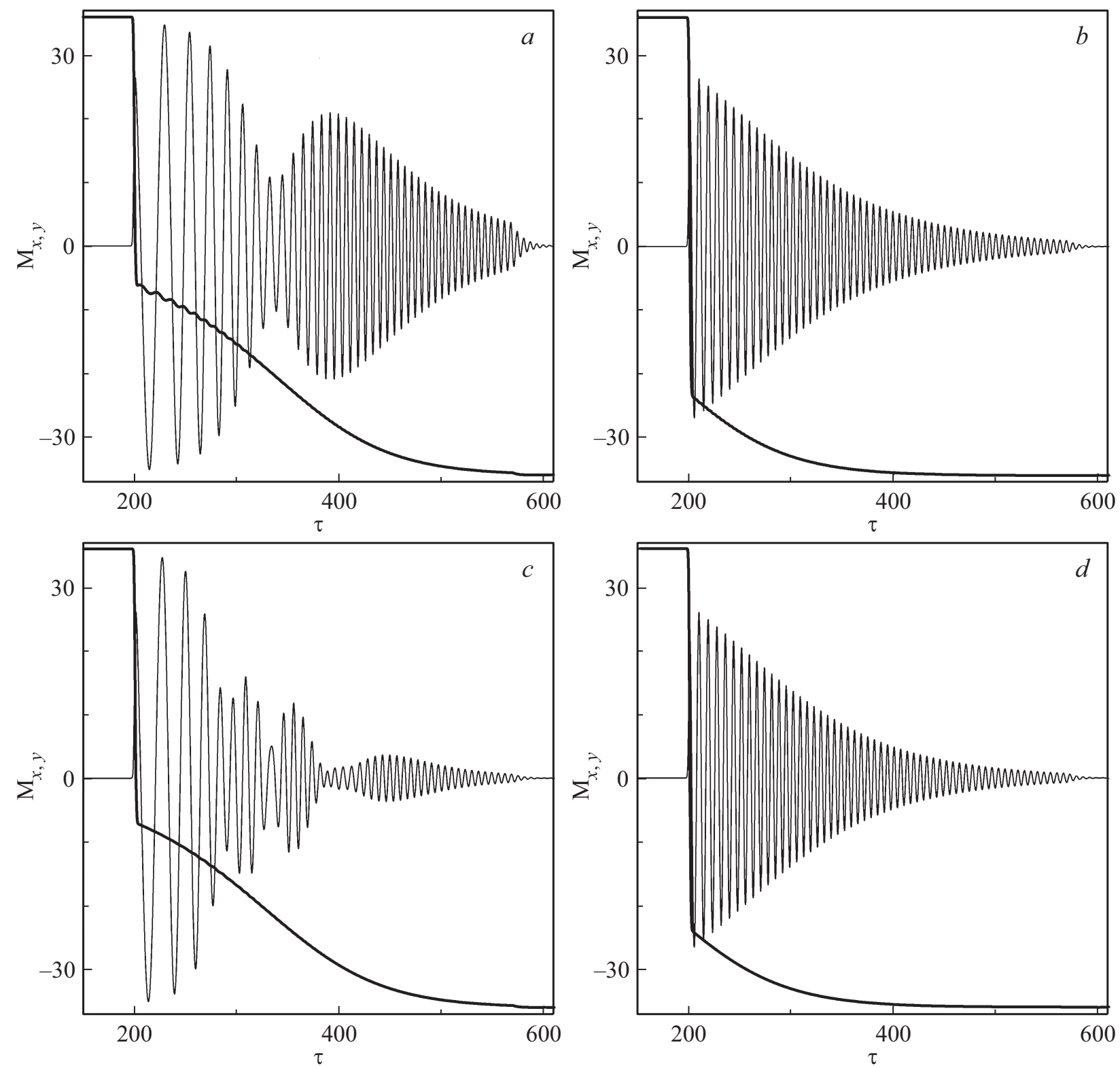

Рис. 8. Зависимость от времени компонент магнитного момента решетки $6 \times 6$ с параметром $p=10$ при перемагничивании импульсом с $\tau_{0}=1, \tau_{i}=200, h_{0}=0.9(a, c)$ и $h_{0}=1.1(b, d)$; в случаях $(a, b)$ ОЛН совпадает с осью $Y$, поле импульса направлено по оси $X$, в случаях $(c, d)$ ОЛН совпадает с осью $X$, а поле импульса - с осью $Y$.

полюса $(a)$. Если магнитный момента оказывается вблизи экваториальной плоскости на стороне противоположного полюса, реализуется продолжительный отклик с перемагничиванием $(d)$. Если же действующий импульс доводит магнитный момент до области вблизи одного из полюсов, отклик - короткий.

\section{5. Отклик решетки магнитных диполей на импульс поля}

Все выявленные особенности импульсного перемагничивания магнитоодноосных наночастиц справедливы также и для решетки при достаточно слабом диполь-дипольном взаимодействии (когда безразмерный параметр решетки $\rho \geq 5$ ). Для решетки наночастиц $6 \times 6$ с параметром $\rho=10$ на рис. 8 приведена зависимость от времени $X, Y$-компонент суммарного магнитного момента $\mathbf{M}=\Sigma \boldsymbol{\mu}_{i}$ при действии гауссова импульса с $\tau_{0}=1$ и $h_{0}=0.9(a, c)$, что соответствует приграничной области интервала перемагничивания, и $h_{0}=1.1(b, d)$, что соответствует области несколько ближе к центру интервала. Для сравнения различных конфигураций в случаях $(a, b)$ принята, прежняя геометрия (ось анизотропии совпадает с осью $Y$ и поле импульса направлено по оси $X)$. В случаях же $(c, d)$ принято, что направление одноосной анизотропии совпадает с перпендикулярной плоскости решетки осью $X$, в исходном состоянии все 

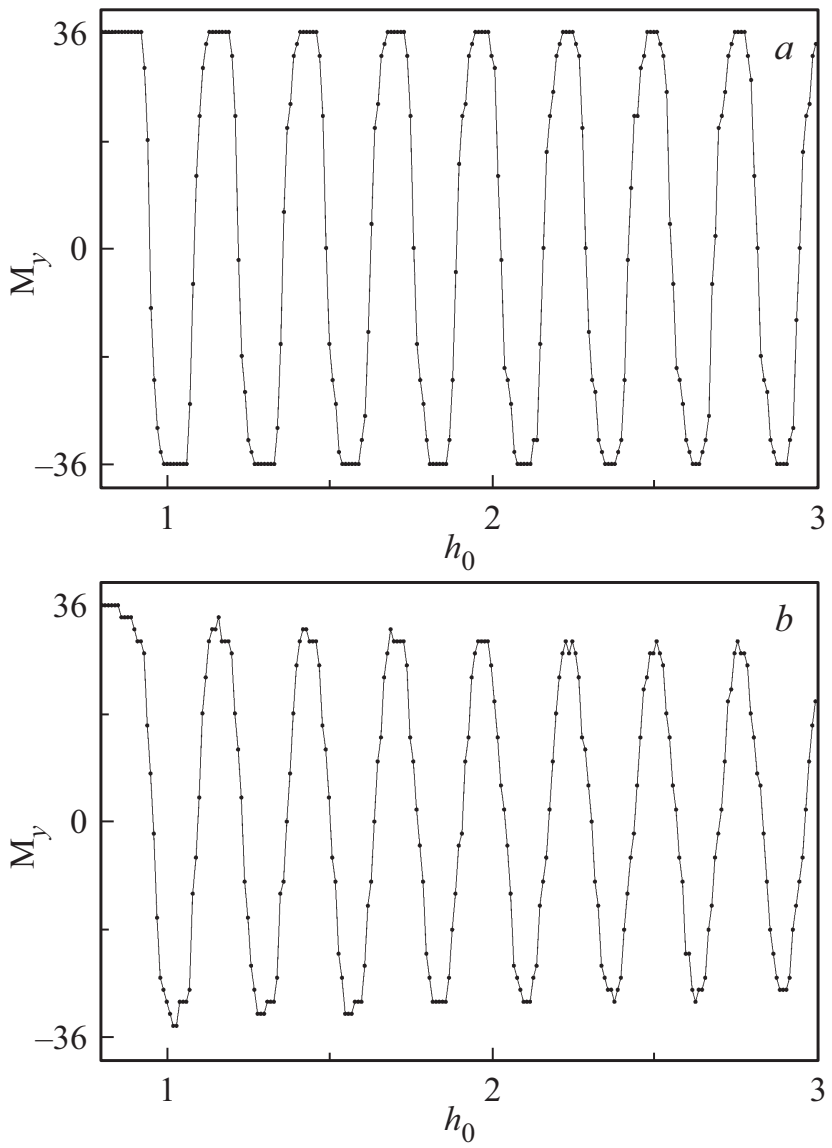

Рис. 9. Диаграммы зависимости равновесного значения $Y$-компоненты магнитного момента решетки $6 \times 6$ с $p=10$ от амплитуды импульса при наличии разброса по углам 0 и $\phi$ ОЛН наночастиц со среднеквадратичным отклонением, равным $1(a)$ и $2(b)$ градуса.

магнитные моменты решетки направлены в положительном направлении оси $X$, а поле импульса ориентировано по оси $Y$. При сравнении данного рисунка с рис. 5 для изолированной наночастицы видно, что слабое диполь-дипольное взаимодействие проявляться возникновением модуляции прецессионного движения. Причем влияние соседних диполей заметно только вблизи границы интервала перемагничивания $(a, c)$ и больше в случае перпендикулярной конфигурации (c). При параметрах импульса, отвечающих центральной области интервалов перемагничивания/неперемагничивания и, соответственно при коротком отклике на действие импульса, влияние диполь-дипольного взаимодействия проявляется только при малых параметрах решетки, когда теряет устойчивость однородная равновесная конфигурация.

В случае наличия разброса ОЛН входящих в решетку наночастиц, относительно задаваемого внешними условиями среднего направления, диаграммы перемагничивания системы изменяются в соответствии величины угловой дисперсии. При этом анализ показывает, что разброс по азимутальному углу $\varphi$, отсчитываемому от оси $Y$ в плоскости решетки $Y Z$ (при рассматриваемой перпендикулярной ориентации поля импульса и при слабом диполь-дипольном взаимодействии), практически не сказывается на прецессионную динамику отклика системы и на ее перемагничивание. Разброс же ОЛН по полярному углу $\theta$, отсчитываемому от плоскости решетки, существенно влияет на отклик и конечную ориентацию отдельных диполей системы, что следует из рис. 4.

На рис. 9 приведены диаграммы зависимости равновесного значения $Y$-компоненты суммарного магнитного момента решетки наночастиц $6 \times 6$ с параметром $\rho=10$ от амплитуды действующего импульса с $\tau_{0}=10$. Диаграммы отвечают нормальному (гауссовому) распределению углов анизотропии по углам $\theta$ и $\varphi$ со среднеквадратичными отклонениями 1 и 2 градуса $(a, b)$. Видно, что в случае $(a)$ между областями полного перемагничивания/неперемагничивания решетки возникают области частичного ее перемагничивания, причем ширина первых сужается с увеличением амплитуды импульса, и при достаточно больших $h_{0}$ полное перемагничивание (и неперемагничивание) будет отсутствовать. При увеличении дисперсии по углам анизотропии $(b)$ полное перемагничивание и неперемагничивание решетки не реализуется, а имеет место близкое к периодическому (с увеличением одного из параметров импульса) изменение числа перемагничивающихся под воздействием импульса наночастиц.

\section{6. Заключение}

Исследование отклика магнитного момента наночастицы с одноосной анизотропией на действие короткого гауссова импульса магнитного поля выявило сильную зависимость реализации перемагничивания и продолжительности прецессионной динамики от длительности и пикового значения импульса. С изменением амплитуды или длительности импульса продолжительность отклика магнитного момента периодически достигает своих максимальных и минимальных значений. При условиях, отвечающих минимуму отклика магнитного момента на действие импульса, после короткого всплеска прецессионной динамики фазовая траектория быстро приближается к равновесному состоянию. Максимумы отклика разбивают область значений параметра импульса на интервалы, отвечающие перемагничиванию наночастицы, которые чередуются с интервалами, отвечающими отсутствию перемагничивания. При этом центральные области интервалов характеризуются коротким откликом магнитного момента, а краевые области - длительным по продолжительности откликом. Ширина данных параметрических интервалов перемагничивания/неперемагничивания сокращается с увеличением длительности импульса или с возрастанием его пикового значения над величиной константы одноосной анизотропии. 
Выявленные особенности отклика наночастицы и решетки на импульсное воздействие обусловлены характером аттракторов прецессионного движения магнитного момента рассматриваемой нелинейной системы. Продолжительность отклика, а также реализация перемагничивания определяются положением магнитного момента относительно оси анизотропии при прекращении действия импульса. В случае модулированного импульса чередование параметрических интервалов перемагничивания/неперемагничивания возникает только при значительном преобладании в импульсе одной полуволны модуляции. В остальных случаях имеют место обширные области бистабильности, когда после действия импульса магнитный момент под влиянием различных флуктуаций может как вернуться к исходному состоянию, так и прийти к противоположной ориентации.

На процессы перемагничивания сильное влияние оказывает подмагничивающее поле, ориентированное вдоль ОЛН наночастиц. С помощью слабого поля можно существенно ограничить области значений параметров импульса, отвечающие либо перемагничиванию, либо неперемагничиванию наночастиц. Аналогичное действие производит отклонение поля импульса на несколько градусов от нормали к ОЛН, причем наиболее сильно на процессы перемагничивания влияет отклонение к направлению, совпадаю- щему с исходной ориентацией магнитных моментов.

В случае решетки наночастиц слабое диполь-дипольное взаимодействие приводит к модуляции прецессионной динамики отклика суммарного магнитного момента. В большей степени это проявляется при перпендикулярной конфигурации, когда легкая ось совпадает с нормалью к плоскости решетки. Однако при обеих конфигурациях влияние диполь-дипольного взаимодействия на прецессионную динамику проявляется только вблизи границ вышеуказанных интервалов параметров импульса. При наличии углового разброса ОЛН отдельных наночастиц решетки, распределенных по гауссовому закону со среднеквадратичным отклонением $\Delta \theta_{i} \geq 1$ градуса, реализуется лишь частичное перемагничивание решетки. При этом с изменением параметров импульса имеет место близкое к периодическому изменение числа перемагничивающихся наночастиц. Полученные результаты выявляют общий характер импульсного перемагничивания дипольных систем с одноосной анизотропией, что подтверждают экспериментальные исследования, проведенные ранее над другими магнитными структурами.

\section{Финансирование работы}

Работа выполнена при поддержке Министерства науки и образования РФ (Госзадание № 3.6825.2017/БЧ) и РФФИ (договор № 18-42-730001/18).

\section{Конфликт интересов}

Авторы заявляют, что у них нет конфликта интересов.

\section{Список литературы}

[1] R. Skomski. J. Phys.: Condens. Matter. 15, R841 (2003).

[2] E.Z. Meilikhov, R.M. Farzetdinova. JMMM 268, 237 (2004).

[3] Ю.П. Иванов, А.И. Ильин, Е.В. Пустовалов, Л.А. Чеботкеич. ФТТ 52, 1576 (2010).

[4] В.А. Кособукин, Б.Б. Кричевцов. ФТТ 52, 759 (2010).

[5] П.В. Бондаренко, А.Ю. Галкин, Б.А. Иванов. ЖЭТФ 139, 1127 (2011).

[6] А.А. Фраерман. УФН 182, 1345 (2012).

[7] С.А. Дзян, Б.А. Иванов. ЖЭТФ 142, 969 (2012).

[8] S.A. Gudoshnikov, B.Ya. Liubimov, A.V. Popova, N.A. Usov. JMMM 324, 3690 (2012).

[9] T. Kiseleva, S. Zholudev, A. Novakova, T. Grigoryeva. Comp. Structures 138, 12 (2016).

[10] M.F. Hansen, P.E. Jonsson, P. Nordblad, P. Svedlindh. J. Phys.: Condens. Matter. 14, 4901 (2012).

[11] А.М. Шутый, Д.И. Семенцов. ФТТ 60, 2369 (2018).

[12] А.М. Шутый, Д.И. Семенцов. Письма в ЖЭТФ 99, 806 (2014).

[13] A.M. Shutyi, S.V. Eliseevay, D.I. Sementsov. Phys. Rev. B 91, 024421 (2015).

[14] A.M. Shutyi, D.I. Sementsov. JMMM 401, 1033 (2016).

[15] N. Eibagi, J.J. Kan, F.E. Spada, E.E. Fullerton. IEEE Magn. Lett. 3, 4500204 (2012).

[16] Е.3. Мейлихов, Р.М. Фарзетдинова. ФТТ 56, 2326 (2014).

[17] H.W. Schumacher, C. Chappert, P. Crozat, R.C. Sousa, P.P. Freitas, J. Miltat, J. Fassbender, B. Hillebrands. Phys. Rev. Lett. 90, 1, 017201 (2003).

[18] H.W. Schumacher, C. Chappert, R.C. Sousa, P.P. Freitas, J Miltat, J. Fassbender. Phys. Rev. Lett. 90, 1, 017204 (2003).

[19] A.V. Kimel, B.A. Ivanov, R.V. Pisarev, P.A. Usachev, A. Kirilyuk, Th. Rasing. Nature Phys. 5, 727 (2009).

[20] Takuya Satoh, Sung-Jin Cho, Ryugo Iida, Tsutomu Shimura, Kazuo Kuroda, Hiroaki Ueda, Yutaka Ueda, B.A. Ivanov, Franco Nori, Manfred Fiebig. Phys. Rev. Lett. 105, 7, 077402 (2010).

[21] А.Ю. Галкин, Б.А. Иванов. Письма в ЖЭТФ 88, 4, 286 (2008).

[22] Ю.И. Джежеря, В.П. Юрчук, К.О. Демишев, В.Н. Коренивский. ЖЭТФ 144, 6(12), 461 (2013).

[23] A. Sukhov, J. Berakdar. Phys. Rev. B 79, 134433 (2009).

[24] В.В. Рандошкин, А.М. Салецкий, Н.Н. Усманов, Д.Б. Чопорняк. ФТТ 46, 3, 2326 (2004).

[25] Е.И. Ильяшенко, О.С. Колотов, А.В. Матюнин, О.А. Миронец, В.А. Погожев. ЖТФ 76, 11, 140 (2006).

[26] Д.А. Балаев, А.А. Красиков, Д.А. Великанов, С.И. Попков, Н.В. Дубынин, С.В. Столяр, В.П. Ладыгина, Р.Н. Ярославцев. ФТТ 60, 10, 1931 (2018).

[27] А.Г. Гуревич, Г.А. Мелков. Магнитные колебания и волны. Наука, М. (1994).

[28] А.М. Шутый, Д.И. Семенцов. Письма в ЖЭТФ 108, 11, 761 (2018).

Редактор Ю.Э. Китаев 